STOCKMANS, F.: Le lignite de Mol. - Bull. Mus. Roy. Hist. Nat., Brüssel 1943.

STOLLEY, E.: Geologische Mitteilungen von der Insel Sylt I. - Arch. Anthrop. Geol. Schlesw.-Holst. 4, S. 3-49, Kiel 1900. - Geologica varia von den Nordseeinseln. - 23. Jahresber. Niedersächs. Geol. Ver., S. 31-111, Hannover 1929.

TAVERNIER, R.: De kwartaire Afzettingen van Belgie. - Natuurwetensch. Tijdschrift, 25, S. 121-137, Gent 1943.

TEN DAM, A. und REINHOLD, TH.: Die stratigraphische Gliederung des niederländischen Plio-Pleistozäns nach Foraminiferen. - Meded. Geol. Stichting, Serie C, V, Nr. 1. Maastricht 1941.

THIELE, S.: Die Stratigraphie und Paläogeographie des Jungtertiärs in SchleswigHolstein. - N. Jahrb. Min. etc. Abt. B., Beil. Bd. 85, S. 1-143, 1941.

THIERGART, F.: Die Mikropalaeontologie als Pollenanalyse im Dienste der Braunkohlenforschung. - Schr. Brennstoffgeol., 13, Stuttgart 1940.

VAN VOORTHUYSEN, J. H.: The Plio-Pleistocene Boundary in the Netherlands based on the Ecology of Foraminifera. - Geologie en Mijnbouw, 12 Jaargang, pp. $26-31,1950$.

WETZEL, W.: Die Sedimenpetrographie des Sylter Tertiärs. - Schr. Naturw. Ver. Schleswig-Holst. 19, S. 204 -233, Kiel 1931. - Neue Beobachtungen am Jungtertiär von Sylt. - 28. Jahresber. niedersächs. geol. Ver., S. 74-104, Hann.1937.

WEYL, R.: Jungtertiäre Schlotten im Zechstein von Lieth bei Elmshorn (mit einer Bemerkung von H. ILLIES). - Schr. Naturw. Verein Schlesw.-Holst., 24, S. $74-81,1949$.

WIRTZ, D.: Die Fauna des Sylter Crag und ihre Stellung im Neogen der Nordsee. Mitteilg. Geol. Staatsinstitut Hamburg, Heft 19, 1949.

WOLDSTEDT, P.: Norddeutschland und angrenzende Gebiete im Eiszeitalter. 464 pp. Stuttgart 1950.

ZEUNER, FR.: The Pleistocene Period. - London 1945.

ZONNEVELD, J. I. S.: Het Kwartair van hat Peel-Gebied en de naaste Omgeving. Meded. Geol. Stichting, Serie C, VI, Nr. 3. Maastricht 1947.

Nachtrag während der Drucklegung

Seit Abschluß des Manuskriptes im Frühjahr 1949 haben P. THOMSON (N. Jb. Min., Abt. B., 1945-48, S. 367-369 und Geol. Jahrb., 65, S. 126), G. KREMP (Geol. Jahrb., 64, S. 511) und P. WOLDSTEDT (Z. dtsch. Geol. Ges. 100, S. 394, 1951) auf pollenanalytische Untersuchungen im Sylter Kaolinsand Bezug genommen. Alle diese Bemerkungen gründen sich indessen, was Sylt betrifft, auf Pollenanalysen THIERGART'S (1940) von Saprohumolithbänkchen im Kaolinsand des Roten Kliffs. Seine Untersuchungen ließen eine altpleistozäne Flora mit wenigen tertiären Relikten erkennen. Im Gegensatz zu WOLDSTEDT muß deshalb betont werden, daß kein grundsätzlicher Widerspruch zwischen der pollenanalytischen und der unsrigen Auffassung vom Alter des Sylter Kaolinsandes besteht, wenn man die Einstufung des Sylter Limonitsandsteins ins höchste Oberpliozän zugrunde legt.

\title{
Untersuchungen an nordwestdeutschen Interglazialen
}

\author{
Von P. Woldstedt, U. Rein und W. Selle. Mit 4 Abb.
}

\section{Vorkommen und Lagerung (P. WOLDSTEDT)}

Bei meinen langjährigen geologischen Aufnahmen in Nordwestdeutschland erhob sich immer wieder die Frage der Gliederung des dortigen Pleistozäns und insbesondere die Frage der Einordnung der Interglaziale. Über das Alter der verschiedenen Interglazialbildungen Nordwestdeutschlands ist auch früher schon viel diskutiert worden. Ich erinnere an die lebhaften Erörterungen über das Alter der Lauenburger Torfvorkommen. Eine erste pollenanalytische Unter- 
suchung nordwestdeutscher Interglazialvorkommen ist durch JESSEN und MILTHERS in ihrem großen, 1928 erschienenen Werk geschehen. Sie glaubten damals zwei Interglazialperioden mit verschiedener Sedimentbildung unterscheiden zu können: eine jüngere Interglazialzeit mit vorwiegender Bildung von Torfen, und eine ältere mit der Bildung von Seekreiden.

Bestimmte Beobachtungen ließen mich an der Richtigkeit dieser Auffassung zweifeln. Ich hielt deshalb eine nochmalige genauere Untersuchung der nordwestdeutschen Interglaziale für dringend erwünscht. Die Preußische Geologische Landesanstalt stellte im Jahre 1936 auf meinen Antrag hin Geld für eine Reihe von Bohrungen zur Verfügung. Es gelang, eine Bohrfirma zu finden, die mit Hilfe eines sog. Stoßkernrohres vollständige, lückenlose Probenserien der Interglazialablagerungen nehmen konnte, und so wurden zusammenhängende Probenfolgen der Interglaziale von Honerdingen, Godenstedt, Mengebostel und Munster gewonnen. Weiter wurden von W. SELLE und U. REIN in mehreren im Abbau begriffenen Mergel- und Kieselgurgruben (u. a. Lehringen, Hützel, Grevenhof, Oberohe, Munster) vollständige Profile von der Basis bis zur Oberfläche genommen.

Ungefähr zur gleichen Zeit hatte W. SELLE mit einer pollenanalytischen Untersuchung des Torfes von Honerdingen sowie der Interglaziale von Gr-Hehlen und Ummendorf begonnen (veröffentlicht 1941). Er und U. REIN nahmen dann die pollenanalytische Untersuchung der gewonnenen Probenserien in Angriff. U, REIN (1938) veröffentlichte eine abgekürztes Diagramm von Lehringen. Ein vollständiges Diagramm von Mengebostel, untersucht von W. SELLE, und ein Teildiagramm von HÜTZEL, untersucht von U. REIN, wurden 1942 in einer Arbeit von mir veröffentlicht. Während des Krieges konnte noch eine Reihe ergänzender Bohrungen ausgeführt und pollenanalytisch von W. SELLE untersucht werden. Im Folgenden sollen die Hauptergebnisse der gemeinsamen Untersuchungen dargelegt werden. Eine ausführliche Behandlung wird demnächst an anderer Stelle erfolgen.

Bei dem Vorkommen handelt es sich zunächst um eine Gruppe von Sü Bw a s s e r m e r g e $1 \mathrm{n}$ : Honerdingen, Lehringen, Neddenaverbergen, Godenstedt, Mengebostel, Neuenförde u. a. Bei diesen Bildungen, ebenso wie bei den später zu besprechenden Kieselgurablagerungen, liegt die Ausfüllung alter Seen, meist glazialer Rinnenseen, vor. Die allgemeine Ausbildung dieser alten Seebildungen (vgl. Abb. 1) ist die, daß meist ein Süßwassermergel oder ein Kieselgurlager (4-10 m mächtig und mehr) den größten Teil der alten Hohlform ausfüllt. Darüber liegt in zahlreichen Fällen ein 1-3 m mächtiger Torf, der die Verlandung

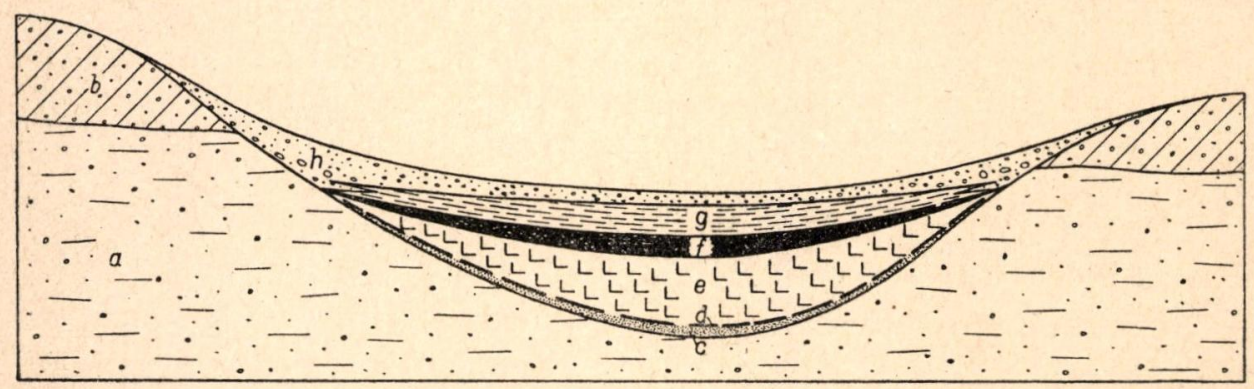

Abb. 1: Schematisches Bild eines Interglazialvorkommens vom sog. Bröruptyp. $\mathrm{a}=$ Sand und Kies („Unterer Sand“). b = Geschiebemergel. c = Unterer Beckensand und -ton. $\mathrm{d}=$ Unteres Torflager. $\mathrm{e}=$ Süßwassermergel oder Kieselgur. $\mathrm{f}=$ Oberes Torflager. $\mathrm{g}=$ Oberer Beckensand und -ton. $\mathrm{h}=$ Geschiebedecksand. 
des ehemaligen Sees anzeigt. Über diesen Interglazialbildungen folgen wieder eiszeitliche Ablagerungen sehr verschiedener Ausbildung und Mächtigkeit. In keinem Falle ist bei einem der oben genannten Interglaziale eine echte Grundmoräne im Hangenden gefunden worden; sondern stets handelt es sich um sandige Bildungen mit mehr oder weniger Geschieben. Verhältnismäßig geringmächtig sind diese Deckschichten z. B. in Lehringen. Dort bestehen sie am Ostrand der Grube aus einem nur 1/2 m mächtigen ungeschichteten Sand mit Kiesen und Geschieben,über dem $40 \mathrm{~cm}$ feiner grauer Sand folgen. Nach Westen, d. h. zum ehemaligen Ufer des Sees hin, werden die Schichten gröber und mächtiger. Ähnlich ist es in Neddenaverbergen, wo ebenfalls in einiger Entfernung vom ehemaligen Seeufer die Deckschichten ganz geringmächtig werden.

Bei einer anderen Gruppe von Interglazialen sind die Deckschichten mächtiger. Meist lassen sich in diesem Fall zwei Abteilungen in den Deckschichten unterscheiden: zu unterst Beckenbildungen, d.h. Bändertone und geschichtete feine Sande, die oft mehrere Meter mächtig werden (Schicht g der Abb. 1). Darüber folgen dann in meist nach dem Rande hin zunehmender Mächtigkeit Geschiebesande (h). Wir haben also als ersten Vorgang nach der Bildung der eigentlichen Interglazialschichten zunächst eine weitere Ausfüllung der vorhandenen Becken, aber nun nicht mehr mit organischen Absätzen, d.h. mit SüßwasserMergel, Torf, Kieselgur usw., sondern mit rein anorganischen, mineralischen Sedimenten. Die Sedimentationsbedingungen müssen sich gegenüber denen der Interglazialzeit grundsätzlich geändert haben.

Wo in der Interglazialzeit die Becken noch nicht ganz ausgefüllt waren, da macht die Vorstellung einer weiteren Ausfüllung in der darauffolgenden Eiszeit keine weitere Schwierigkeit. Wo aber die Ausfüllung der Hohlformen bis zum ehemaligen Wasserspiegel schon in der Interglazialzeit beendet war - und das war der Fall, wo Torfbildungen den Mergel oder die Kieselgur überlagern da erscheint es zunächst schwer verständlich, wie eine nochmalige Überflutung des Beckens zustande kam. Solche Verhältnisse liegen z. B. im Falle Honerdingen vor. Dort bedeckt ein bis sechs Meter mächtiger Beckensand den Torf.

Das schematische Profil (Abb.1), das auf Grund eigener Beobachtungen in Honerdingen und Lehringen sowie unter Berücksichtigung der früheren Bearbeitung Honerdingens durch C. A. WEBER (1896) gezeichnet wurde, läßt aber erkennen, daß diese Beckensande, wie der unterlagernde Torf, muldenförmig gelagert sind und daß sie in der Mitte des Beckens ihre größte Mächtigkeit haben. Auch die Torfschicht erreicht hier ihre größte Dicke, die nach C. A. WEBER $3^{1 / 2} \mathrm{~m}$ beträgt. Muldenförmige Lagerung und Zunahme der Mächtigkeit von Torf und Beckensanden müssen ihre Ursache wohl hauptsächlich in Schrumpfungen des unterlagernden Mergels haben ${ }^{1}$ ). Diese begannen schon in der Interglazialzeit, wie die nach der Mitte zunehmende Mächtigkeit des Torfes zeigt. Sie setzte sich während der darauffolgenden (letzten) Eiszeit fort. Der Torf sank allmählich unter den Wasserspiegel. Zugleich wurde von den seitlichen Höhen sandiges Material eingeschwemmt, das sich schließlich in beträchtlicher Mächtigkeit über der immer noch nachsackenden Unterlage anhäufte. Beim Torf spielte dabei auch die Zusammendrückbarkeit unter der Last des Sandes eine Rolle. Schließlich aber waren die Sackungen beendet und war der über der alten Hohlform neu entstandene See zugefüllt.

$\mathrm{Ob}$ in einzelnen Fällen vielleicht auch dadurch die alten Hohlformen neu geschlossen wurden, daß Abdämmungen durch Fließerden und dgl. stattfanden,

1) Auf entsprechende Schrumpfungen bei nacheiszeitlichen Wiesenmergeln machte mich Prof. K. RICHTER freundlicherweise aufmerksam. 
oder ob eine Hebung des Wasserspiegels im Zusammenhang mit einem Frostboden in der höheren Umrandung eintreten konnte, bedarf noch näherer Untersuchung.

Über den Beckenbildungen finden wir dann als zweite Schicht, wie schon gesagt wurde, meist Geschiebesande. Sie wurden zweifellos subaërisch abgelagert, d. h. sie wanderten von den Seiten herein, nachdem die Becken bis zum Wasserspiegel ausgefüllt waren. Häufig sind vom Rande der Becken her alle Ùbergänge von echter Grundmoräne über Pseudogrundmoränen zu Geschiebesanden vorhanden. Über dem Hauptteil der Interglaziale, und zwar sowohl der M e r g e 11 a g e r der Lüneburger Heide wie der gleich zu besprechenden K i e selgurlager, ist noch in keinem Falle je echter Geschiebemergel gefunden vorden. Randlich tritt gelegentlich Geschiebemergel auf; aber in allen Fällen steht nicht ganz fest, ob es sich um anstehenden echten Geschiebemergel handelt oder um umgelagerten.

Was nun die Kieselgurlager anbelangt, so gilt für die Deckschichten ganz dasselbe wie für die Mergellager. Die Kieselgurlager treten, wie die Mergellager, sowohl im Bereich des Warthestadiums wie außerhalb von diesem auf. Die wichtigste Gruppe von Vorkommen in n e $\mathrm{h}$ a $1 \mathrm{~b}$ des Warthestadiums liegt im oberen Luhetal. Hier sind die Deckschichten vielfach nur ganz geringmächtig, besonders wenn die Vorkommen mehr in der Mitte des Tales belegen sind.

Für die Altersverhältnisse der Vorkommen im Luhetal ergibt sich folgendes: Die Kieselgurvorkommen stellen die Ausfüllung einer Seenkette dar, die im oberen Luhetal während des Warthestadiums subglaziär gebildet wurde. Hier war ein Tunneltal vorhanden, das aus zahlreichen, durch Schwellen getrennten, hintereinander liegenden Becken bestand und an der Hauptrandlage des Warthestadiums endete. Das Alter dieses Tunneltals liegt also fest: es ist warthestadial. Es steht weiter fest, daß dies Gebiet nicht nochmal wieder vom Eis bedeckt worden ist. Die Kieselgurvorkommen gehören also eindeutig in die nach dem WartheStadium folgende Interglazialzeit, $d . h$. in die letzte. Damit stimmen die Pollendiagramme, soweit sie aus dem oberen Luhetal gemacht worden sind, aufs beste überein.

Es ergibt sich aber noch eine weitere wichtige Tatsache: Die Kieselgurvorkommen zeigen sich auch im oberen Luhetal teilweise stark gestört. Einzelne Partien erscheinen gegenüber anderen verschoben. Vielfach sind auch größere Teile zu Sätteln und Mulden aufgestaucht. Da ein Gletscher die Lager nicht mehr überschritten hat, kann das Eis nicht für diese Störungen verantwortlich gemacht werden. Die Ursache muß in anderen Kräften gesucht werden, d. h. vielleicht in Gleitbewegungen während eines noch nicht ganz verfestigten $\mathrm{Zu}-$ standes; Gleitbewegungen, die minstens zum Teil mit einer ungleichmäßigen Belastung durch die Deckschichten zusammenhängen. Es zeigt sich auch als durchgehende Gesetzmäßigkeit, daß die Störungen um so größer werden, je mächtiger die Deckschichten sind. Für die Altersbestimmung der außerhalb des Warthestadiums liegenden Kieselgurvorkommen ergibt sich aus den eben gemachten Darlegungen, daß die Störungen in diesen Kieselgurlagern nicht als Beweis für eine nochmalige Eisbedeckung angeführt werden können.

Ich komme damit zum Alter der außerhalb des Warthestadiums gelegenen Kieselgurvorkommen. Wenn auch, wie eben gesagt wurde, aus den Stauchungen der Kieselgurlager nicht auf eine nochmalige Eisbedeckung geschlossen werden kann, so darf doch nicht ohne weiteres gesagt werden, eine solche Eisbedeckung habe sicher nicht stattgefunden. Würden die Kieselgurlager außerhalb des Warthestadiums dasselbe Pollendiagramm zeigen, wie die im oberen Luhetal 
und wie fast alle bisher untersuchten Süßwassermergel, dann könnten wir mit ziemlicher Sicherheit sagen, daß auch die Kieselgurvorkommen von Oberohe und Munster in die letzte Interglazialzeit (Saale-Weichsel) gehörten. Die Pollendiagramme von Munster und Oberohe sind aber nicht dieselben wie die des oberen Luhetals, sondern weisen wesentliche Unterschiede auf (vgl. die Ausführungen von W. SELLE).

Theoretisch müssen wir mit der Möglichkeit rechnen, daß wir in der Lüneburger Heide Kieselgurbildungen verschiedenen Alters haben können. Ob es in einem See zur Kieselgur- oder Süßwassermergelbildung kam, hing von der Natur der umgebenden Landschaft ab. War diese vorwiegend aus kalkhaltigen Schichten aufgebaut, z. B. aus frischer kalkhaltiger Grundmoräne, so kam es zum Absatz von Mergeln. Waren es aber mehr Sande, so wurden nach Verbrauch der geringen Kalkmengen die kieseligen Gurbildungen abgesetzt. Vorwiegend sandige Umgebung kann sowohl während der Elster-Saale-Interglazialzeit wie in der Saale-Weichsel-Interglazialzeit in der Lüneburger Heide vorhanden gewesen sein.

Alles in allem genommen, sprechen aber die Lagerungsverhältnisse doch eher für ein letztinterglaziales Alter auch der Kieselgurvorkommen von Ohe und Munster, als für eine Zugehörigkeit zur Elster-Saale-Interglazialzeit. Für die Zuordnung dieser Kieselgurvorkommen zu einer besonderen Saale-WartheInterglazialzeit liegen keine Anzeichen vor. Es bedarf weiterer Untersuchungen über die merkwürdigen Pollendiagramme von Ohe und Munster, deren genaue stratigraphische Stellung also noch offen bleiben muß.

Ein kurzes Wort noch über die vielfach die Interglazialbildungen unterlagernden Schichten. In mehreren Fällen, so z. B. in Lehringen, fand sich unter dem Süßwassermergel nochmals wieder eine Torfschicht von allerdings geringer Mächtigkeit. Sie liegt heute in der Mitte des Beckens tief unten und findet sich auch mehr oder weniger zusammenhängend an den ehemaligen Ufern des Sees (Abb. 1, Schicht d). Die Torfschicht enthält nach einer vorläufigen Untersuchung von S. SCHNEIDER u. a. Betula nana, Birken- und Kiefernholz, dagegen außer Knospenschuppen von Populus tremula keine Laubbäume. Die Torfschicht kann in dieser Form nicht abgelagert sein, sondern muß früher annähernd horizontal gelegen haben. Unter dem Torf findet sich Sand und Bänderton. Wir haben hier also ganz ähnliche Ablagerungen, wie sie in klassischer Weise von MEIENDORF beschrieben worden sind (vgl. RUST, GRIPP und SCHÜTRUMPF 1937). In der Hohlform des Sees lag zunächst ein Toteisklotz. Er wurde unter Sand und Schutt begraben. Nur ganz langsam schmolz er ab, und es wurden Bändertone und Sand über ihm abgelagert. Schließlich kam es zu einer geringen Torfbildung an der damaligen Oberfläche. Dann aber begann die interglaziale Wärmezeit zu wirken, und nun taute das begrabene Toteis sehr schnell auf, die Torfschicht sank herunter oder legte sich in Fetzen auf die seitlichen Ufergehänge. Der eigentliche tiefe Rinnensee entstand erst jetzt. Er wurde allmählich mit Süßwassermergel angefüllt, bis das Wasser so seicht war, daß sich die obere Torfschicht bilden konnte.

Ähnliche Bildungen dürften auch bei anderen Interglazialen zu finden sein. Es ist bisher auf diese Dinge verhältnismäßig wenig geachtet worden. Aber auch STOLLER (1910) erwähnt von Neddenaverbergen eine Sumpfschicht von der Basis des Mergels. Hier liegt offenbar eine ähnliche Bildung vor wie in Lehringen. Im übrigen reichten die Seen verschieden tief in ältere Ablagerungen 


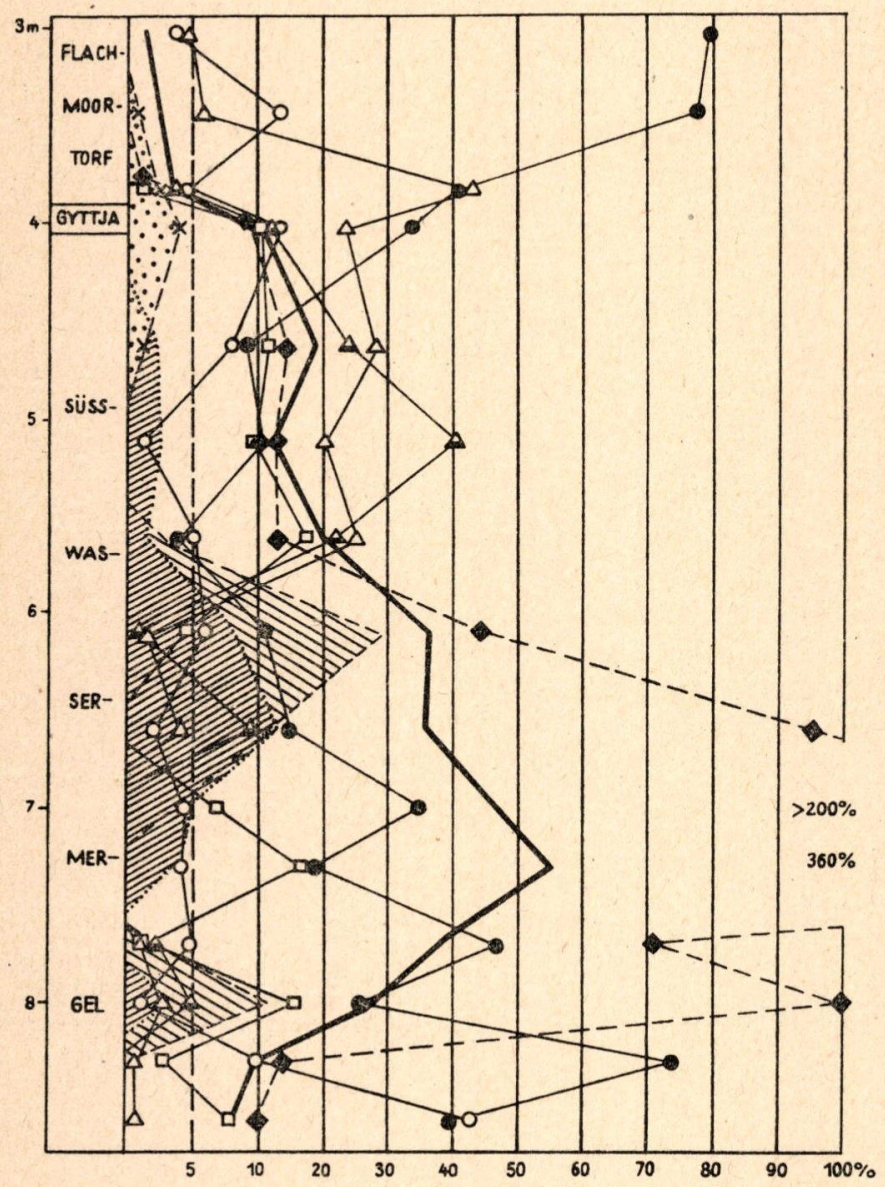

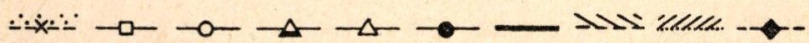

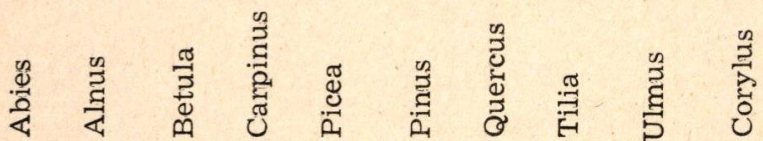

Abb. 2: Abgekürztes Pollendiagramm des interglazialen Süßwassermergels von Godenstedt bei Zeven. herunter, meist in die sog. Unteren Sande, d. h. Sande, die unter der Saale- bzw. WartheGrundmoräne liegen (a der Abb. 1).

Aus der großen Reihe von Einzeluntersuchungen seien im Folgenden einige wenige kurz behandelt. Das von U. REIN bereits 1938 kurz beschriebene Vorkommen von Lehringen hat inzwischen dadurch ein besonderes Interesse erhalten, als dort 1948 im Süßwasser-Mergel das fast vollständige Skelett eines Elephas antiquus zusammen mit einer $2 \frac{1}{2} \mathrm{~m}$ langen Stoßlanze aus Eibenholz sowie mit Steinwerkzeugen gefunden wurde. Eine monographische Bearbeitung des Mergellagers Lehringen mit seinen verschiedenen Funden ist seitens des Landesmuseums Hannover geplant. In diesem Zusammenhange wird U. REIN eine eingehende Behandlung der Vegetationsentwicklung auf Grund neuer Untersuchungen geben.

2. Die Vegetationsentwicklung in der Interglazialablagerung von Godens ted t b e i $\mathbf{Z}$ even (U. REIN)

Große Ähnlichkeit mit dem 1938 von mir beschriebenen Vorkommen von Lehringen weist sowohl in den Lagerungsverhältnissen als auch in der Vegetationsentwicklung während seiner Ablagerung der Süßwassermergel von Godenstedt bei Zeven auf. Auch in ihm ist der bekannte Wechsel des Klimas und der Vegetation, deren Blütenstaub während der Auffüllung der Senke mit Mergel in diesen hineingeweht wurde, zu erkennen. Unter einer $2 \mathrm{~m}$ mächtigen Schicht von Dünen- und Geschiebesanden liegen $2 \mathrm{~m}$ Torf, eine dünne Faulschlammschicht und 4,6 m Süßwassermergel. 
Von den 70 untersuchten Proben wurden 16 zur Darstellung der Entwicklung in einem Übersichtsdiagramm (Abb. 2) ausgewählt. In den untersten Proben ist єin Maximum von Kiefer und Birke zu erkennen. Während der Anteil der Birke sehr rasch zurückgeht und erst in den obersten Proben wieder an Bedeutung gewinnt, gibt die Kiefer nur zögernd Raum und hat auch zum Ausgang der Interglazialzeit schon früher wieder ihre Herrschaft angetreten. Zwischen diesen beiden Maxima liegt der Höhepunkt der Eichenmischwaldzeit. Neben dem frühen und raschen Anstieg der Eichenwerte ist gleichzeitig ein großes Maximum der Hasel zu beobachten. Im oberen Abschnitt des Profils klingen beide Arten gemeinsam aus. Die Ulme und die Linde lassen einen ersten Vorstoß und daran anschließend eine Blütezeit erkennen. Die Hainbuche und die Fichte treten fast gleichzeitig und sehr früh auf, sind aber oberhalb der Eichenmischwaldzeit nacheinander die wichtigsten Repräsentanten von Vegetationsgemeinschaften. Zunächst erreicht die Hainbuche nach dem Maximum von Eiche und Linde ihren Höhepunkt in der Verbreitung, und daran anschließend ist die Fichte mit größten Werten vertreten. Gleichzeitig ist die Tanne in Erscheinung getreten, die dann zum Ausgehenden des Interglazials hin verschwindet.

Somit lassen sich in diesem Profil folgende Vegetationsphasen erkennen (von oben nach unten):
VI. Kiefern-Tannen-Birkenzeit
V. Fichten-Tannenzeit
IV. Hainbuchenzeit
III. Eichenmischwaldzeit
II. Erlen-Kiefern-Eichenmischwaldzeit mit Haselgipfel
I. Birken-Kiefernzeit

Das Pollendiagramm läßt eine weitgehende Übereinstimmung der Vegetationsentwicklung mit der von weiteren bekannten letztinterglazialen Ablagerungen erkennen. Das Mergellager von Godenstedt liegt auf Ablagerungen der Saaleeiszeit und ist offensichtlich nicht mehr von einem jüngeren Eisvorstoß überfahren worden. Bei den hangenden Sanden handelt es sich, abgesehen von den Dünen, wahrscheinlich um periglazial abgelagerte Geschiebesande.

Wenngleich die Vegetationsentwicklung in den Ablagerungen von Godenstedt identisch zu sein scheint mit derjenigen, die aus dem unmittelbar vor der Weichseleiszeit liegenden Interglazial bekannt ist, so bedarf es nach wie vor der Klärung, ob in gleich alten interglazialen Ablagerungen die im Pollendiagramm erkennbare Vegetationsentwicklung immer gleichartig sein wird und ob in verschieden alten Ablagerungen die Entwicklung verschieden sein muß. Das Wechselspiel von Großklima und ökologischer Standortsbedingung in Auswirkung auf die Vegetation scheint mir für die Zwischeneiszeiten noch nicht so hinreichend geklärt zu sein, als daß eine interglaziale Bildung bei ungeklärten Lagerungsverhältnissen in jedem Fall allein aufgrund des Pollendiagramms stratigraphisch eingeordnet werden kann. In diesem Sinne müssen auch die Unterschiede zwischen Lehringen (kleiner Haselgipfel und sehr spätes Eichenmaximum) und Godenstedt erneut untersucht werden. Eine Klärung kann nur von stratigraphisch gesicherten Profilen her erfolgen.

\section{Zusammenfassende Betrachtungen über die Vegetationsentwicklung bei den verschiedenen Interglazialvorkommen (W. SELLE)}

Von H o n e r d i n g e n, das durch C. A. WEBER (1896) bekannt geworden ist, wurden bereits zwei Pollendiagramme veröffentlicht, die an Aufschlüssen der 


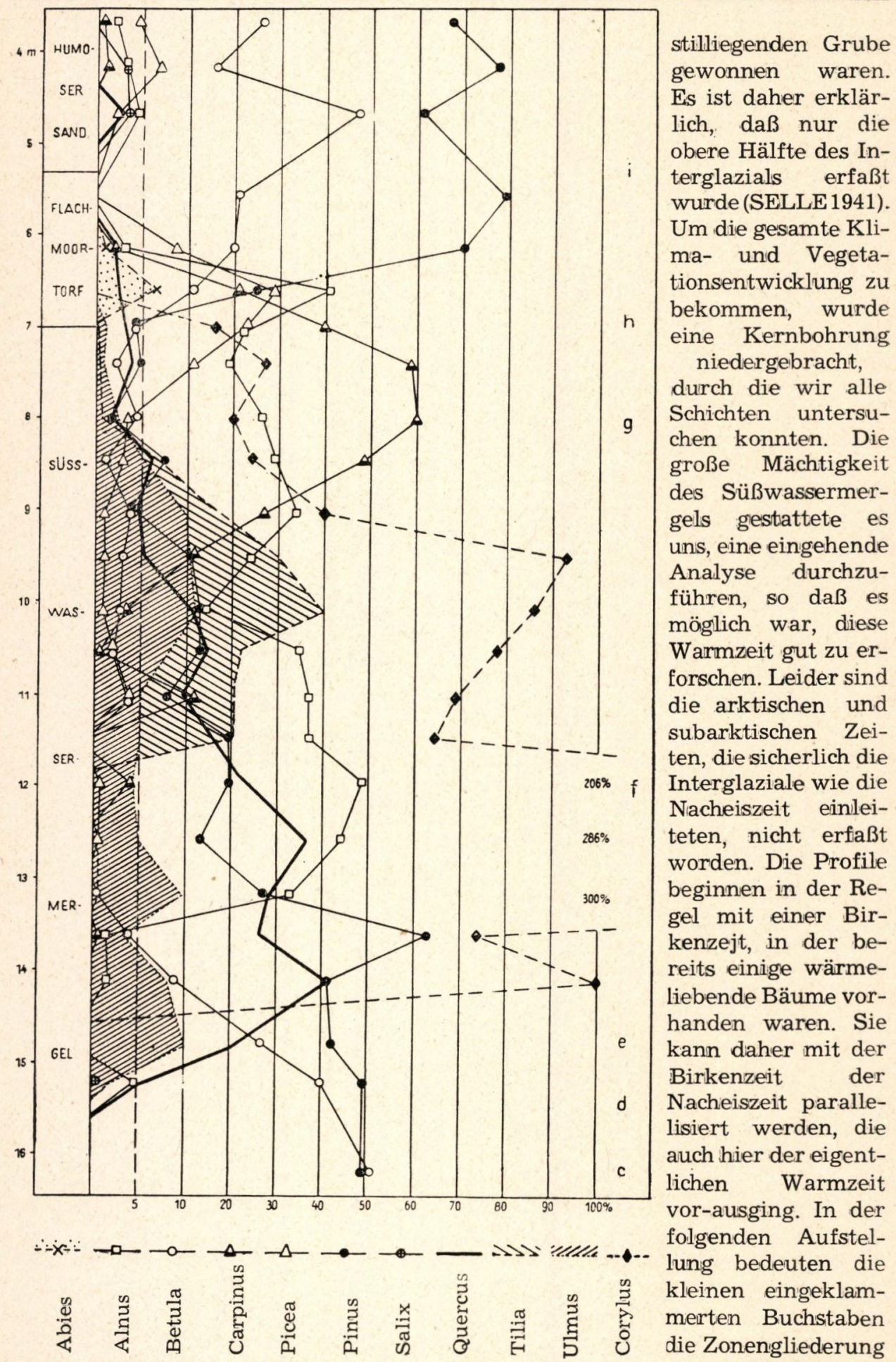

Abb. 3: Abgekürztes Pollendiagramm von Honerdingen III. von JESSEN und 
MILTHERS (1928), während die römischen Ziffern unsere Einteilung wiedergeben, die infolge der eingehenderen Untersuchungen weiter differenziert werden mußten. Aus diesem Grunde war es nicht zweckmäßig, an der Gliederung von JESSEN und MILTHERS festzuhalten (vergl. die stark vereinfachte Abb. 3, die nicht alle Einzelheiten hervortreten läßt).

III (c): Birkenzeit: Betula-Pollen herrschen vor; Kiefer daneben stärker vertreten; wärmeliebende Bäume sporadisch vorhanden; Picea-Pollen vereinzelt; die Werte der NBP (Kräuterpollen) deuten auf einen fast geschlossenen Baumbestand; beginnende Wärmezeit, Klima kontinental.

IV (d): Kiefern-Birkenzeit: Betula und Pinus in der Regel gleich, Pinus jedoch des öfteren dominierend; Eiche und Ulme wandern ein; Picea-Pollen sporadisch; NBP deuten auf geschlossene Wälder; Klima warm-kontinental.

$\mathrm{V}$ (e): Ki f e r n-E M W - Z e i t: Kiefernkurve dominierend; Eiche gewinnt rasch an Bedeutung und hat mit etwa $40 \%$ ihr erstes Maximum; Ulmenkurve durchgehend vorhanden, $10 \%$ aber nicht übersteigend; AlnusPollen vereinzelt; empirische Pollengrenze von Corylus am Ende dieses waldgeschichtlichen Zeitabschnittes; Betula-Kurve stark sinkend, ihre Werte liegen durchschnittlich zwischen 10 und $20 \%$; Klima warmkontinental.

VIa (f): Ki e fer $\mathrm{n}-\mathrm{EM} \mathrm{W}--\mathrm{H}$ a s e $1 \mathrm{z}$ e it: Haselkurve rasch ansteigend, ihre Werte liegen zwar hoch, aber gewöhnlich noch unter $100^{\circ} \%$; zweites Maximum der Eiche um 40\%; Kiefer etwas abnehmend; Birke verliert weiter; Ulmus behält ihre Werte; Linde gering vertreten; Erlenkurve wird zusammenhängend; Picea-Pollen vereinzelt; Klima warm mit langsam wachsender atlantischer Tönung.

VIb (f): EM W-Has elzeit: Die Haselkurve erreicht mit über $300 \%$ ihre höchsten Werte; die Eiche hat ihr drittes Maximum am Ende dieses Abschnittes, ihre Kulmination liegt jedoch etwas tiefer als vorher; die Ulmenkurve sinkt etwas; die Linde erhält ihre empirische Pollengrenze; Hainbuche und Fichte sporadisch vertreten; die Erlenwerte steigen an und erreichen mit ca. $50 \%$ ihren höchsten Stand; Kiefernkurve fällt weiter; Klima warm-atlantisch.

VIc (f): Linden-H a selzeit: Haselkurve sinkt unter $100 \%$, bleibt aber noch dominierend; Linde kulminiert mit ca. 40\%; Ulme erreicht mit gut $10 \%$ ebenfalls ihre höchsten Werte; Eiche verliert an Bedeutung; die abnehmende Tendenz der Kiefer ist weiter vorhanden; Hainbuche und Fichte wandern ein; die Erlenkuve liegt etwas tiefer als zur EMWHaselzeit; Birke weiterhin gering beteiligt; Höhepunkt der Wärmezeit, feucht-warm.

VIIa (g): Hainbuchenzeit: Spontane Ausbreitung der Hainbuche, ihre Kulmination befindet sich bei ca. $60 \%$; Hasel- und Lindenkurve nehmen stark ab; Eichen- und Ulmenwerte im Durchschnitt unter 10\%; die Erle hat einen ähnlichen Stand wie zur Linden-Haselzeit; die Kiefer erreicht ihre geringste Beteiligung; Fichte gewinnt an Bedeutung; Klima kühlatlantisch.

VIIb (g): $\mathrm{H}$ a in buchen-Fich tenzeit: Fichte steigert ihre Werte, während die Hainbuchenkurve sinkt. Die Pollenbilder dieses Zeitabschnittes, wie auch die von VIIa und VIII sind oft durch die Torfe stark gestört. Die Hainbuchenwerte treten in den Süßwassermergeln gut in Erscheinung, in den Torfen dagegen kann die Fichte bereits während 
der Zone VIIa stark überrepräsentiert werden. Sie stockte in den Bruchwaldmooren, wodurch ihr Pollenniederschlag das Diagramm stark beeinflußt.

VIII (h): Fichtenzeit: Die Mergelablagerungen klingen in diesem Zeitabschnitt aus und werden von Torfen abgelöst. Wie bereits erwähnt, beginnen die Bruchwaldbildungen zum Teil schon in der Hainbuchenzeit. Die Süßwassermergel spiegeln ebenfalls die Klimaänderungen wider, so daß bereits an den Ablagerungen die waldgeschichtlichen Zeiten deutlich in Erscheinung treten, so sind z. B. die Kalkgyttjen der Hainbuchenzeit dunkel gefärbt. Wo die Mergelablagerungen spät ausklingen, tritt die Fichtenzeit nur kurz in Erscheinung, während in den Torfen die Fichte sehr hohe Werte erreicht, die sicherlich die Beteiligung von Picea stark überbetonen.

Die Tanne wandert ein, und die wärmeliebende Flora nimmt langsam ab. Erlenkurve sinkt; Eiche unter $10 \%$; Ulme und Linde verlieren ihre zusammenhängende Kurve; Klima kühl-atlantisch.

IX (h): Fichten-Kiefern-Tannenzeit: Tanne kulminiert mit ca. $10 \%$; Fichtenkurve sinkt; Kiefernwerte steigen schnell an und werden dominierend; Birke gewinnt; Eiche unter 10\%; Ulme und Linde sporadisch; Hainbuche nur einige Prozente; Erlenkurve oft unter 10\%; Klima montan, kühle Sommer, aber verhältnismäßig milde Winter.

X (i): Kief ernzeit: Kiefer dominierend; Eiche und Erle verlieren ihre zusammenhängende Kurve; Birke steigert weiter ihre Werte; Hainbuche sporadisch; Salix gering vertreten; Fichte in der Regel unter 10\%; NBP nehmen zu und zeigen die Auflockerung der Wälder an (subarktische Steppe); Klima subarktisch.

Charakteristisch für diese Interglazialzeit sind die scharf abgegrenzten waldgeschichtlichen Phasen, in denen in der Regel die betreffenden Bäume sich spontan ausbreiten. Es folgen so aufeinander vom Anfang bis zum Ende der Warmzeit: Birke, Kiefer, Eiche, Hasel, Linde, Hainbuche, Fichte und Kiefer. Die Süßwassermergel verändern sich in ihrer Zusammensetzung (Farbe, Gewicht, Bestandteile) und Mächtigkeit mit den Waldphasen. Besonders auffallend ist der Wechsel mit dem Beginn der Hainbuchenzeit, die teilweise die Torfbildung einleitet. Mit der Fichtenzeit kommt die Ablagerung des Süßwassermergels zum Erliegen. Die Torfe, die auf die Kalkgyttjen folgen, ändern wiederum ihre Zusammensetzung mit den waldgeschichtlichen Zeiten. Waren es anfangs Flachmoor- und Bruchwaldbildungen, so folgen ihnen mit der Fichten-Kiefern-Tannenzeit, bzw. der Kiefernzeit meso- bis oligotrophe Ablagerungen, die z. T. immer stärker mit Sand durchsetzt werden. Das Klima prägt sich nicht nur in der Florenzusammensetzung aus, sondern ist auch bestimmend für die Ablagerungen in den Becken.

Ein weiterer gemeinsamer Zug ist ihr Auftreten in Talniederungen; so liegt Godenstedt im Talgebiet der Oste, Neddenaverbergen-Lehringen im Lehrde-Tal, Mengebostel und Honerdingen im Böhme-Tal und die Kieselgur-Vorkommen von Schwindebeck, Grevenhof, Steinbeck, Hützel und Hützel-Bispingen, die ebenfalls die oben beschriebene Waldentwicklung haben, im Luhe-Tal. Ihre Becken dürften demnach Ausstrudelungen in Schmelzwasserrinnen,-also subglaziärer Entstehung sein (SELLE 1947).

Eine ähnliche Waldentwicklung weisen die Pollendiagramme auf, die JESSEN und MILTHES (1928) als Brörup-Typ bezeichnet haben, und die sie ebenfalls in die letzte Interglazialzeit stellen. In Nordwestdeutschland haben Godenstedt, 
Lehringen (REIN 1938), Mengebostel (SELLE in Woldstedt 1942), Nedden-Averbergen, Örrel, Lauenburg (SCHÜTRUMPF 1937) und Römstedt (JESSEN und MILTHERS 1928) dieselben waldgeschichtlichen Phasen, und aus Schleswig-Holstein, Ostdeutschland und Polen sind Oldenbüttel (HECK 1932), Rinnersdarf (STARK, FIRBAS und OVERBECK 1932), Elbinger Yoldienton, Dzbanki (PIECH 1932), Poniemun (DYAKOWSKA 1936) und Samostrzelniki (TRELA 1935) in die gleiche Zeit einzureihen, so daß es unter der nötigen Vorsicht möglich ist, einige regionale Unterschiede herauszuschälen. Linde und Tanne weisen z. B. allem Anschein nach ein Ostwestgefälle auf, wie aus der folgenden Aufstellung hervorgeht:

$\begin{array}{lcc} & \text { Linde } & \text { Tanne } \\ \text { Godenstedt } & 28 \% & 9 \% \\ \text { Nedden-Averbergen } & 32 \% & \\ \text { Honerdingen } & 40 \% & \mathbf{8 \%} \\ \text { Rinnersdorf } & 63 \% & 42 \% \\ \text { Poniemun } & 56 \% & \\ \text { Dzbanki } & & 90 \% .\end{array}$

Die geringe Beteiligung der Tanne bei Oldenbüttel $(8 \%)$ und in dem Elbinger Yoldienton $(3 \%)$ ist vermutlich auf die Ausdehnung des Eem-Meeres zurückzuführen, wodurch der atlantische Einfluß bis nach Ostpreußen reichte, während das Binnenland Ostdeutschlands und Polens ein kontinentales Klima besaß. Selbstverständlich bedürfen diese Schlüsse noch weiterer Untersuchungen, bevor sie als gesichert angesehen werden können.

Viel ungenauer sind bislang unsere Kenntnisse über die älteren Interglaziale. Da sie der Erdoberfläche nicht so nahe liegen, wie das bei den jüngeren Interglazialvorkommen der Fall ist, kann es nicht anders erwartet werden. Über die Kieselgurlager von $\mathrm{Munst}$ e $\mathrm{r}$ (Abb. 4) und $\mathrm{Obe} \mathrm{r}-\mathrm{O}$ h e gehen die Ansichten stark auseinander, was beweist, daß wir im Augenblick nicht in der Lage sind, sie eindeutig in die pleistozäne Schichtenfolge einzugliedern. GISTL (1928) hat umfangreiche Pollenanalysen von der Neu-Oher Kieselgur ausgeführt, nach denen er folgende Waldphasen aufstellte (von unten nach oben):
1. Kiefern-Birkenzeit
2. Kiefern-Haselzeit (Hasel $38 \%$ )
3. Hainbuchenzeit (Hainbuche 19\%)
4. Fichtenzeit (Fichte $22 \%$ )
5. Kiefernzeit.

Die Kiefern-Birkenzeit am Anfang der Ablagerungen und die Kiefernzeit im Hangenden zeigen, daß das gesamte Interglazial erfaßt ist. Wenn wir von diesen beiden Waldphasen absehen, können wir feststellen, daß das Pollendiagramm eine große Gleichförmigkeit aufweist. Kiefer und Erle sind dominierend, wodurch die anderen Baumpollenwerte stark zurückgedrängt erscheinen. Eine weitere charakteristische Erscheinung ist die durchgehende Beteiligung der Fichte und Hainbuche, wenn wir von der Birke und den EMW-Bildnern absehen, die auch im Honerdinger Typ fast während des gesamten Interglazials auftreten. Allerdings weisen sie hier eine differenzierte Beteiligung auf, die der EMW bei Neu-Ohe vermissen läßt. Die aufeinanderfolgenden Kulminationen von Hasel, Hainbuche und Fichte, die zwar außer der Hasel nur schwach sind, lassen vermuten, daß die Profile nicht durch Rutschungen getrübt sind, wie sie z. B. im Kieselgurlager von Grevenhof im Luhetale nachgewiesen werden konnten. Die plastischen Kieselgursedimente geraten bei einer steilen Beckenwand leicht ins Gleiten, so daß diese Möglichkeit bei der Beurteilung von Kieselgurprofilen zur Vorsicht mahnt. 
Die Bänderung der Gur bei Ober-Ohe und Munster ließ die Möglichkeit zu, die Zeitdauer des Interglazials zu errechnen. Beide Läger haben ergeben, daß die Warmzeit etwa 10-12000 Jahre umfaßte (vergl. GIESENHAGEN 1926). Bislang wurde angenommen, daß die dunklen Sedimente im Winter zum Absatz kamen, während die helleren ein Niederschlag des Sommers seien. Beide zusammen umspannen einen Jahreszyklus, wie es auch bei den Warwen der Fall ist. Bei den Sedimenten der Eisstauseen mußten die dunklen Streifen im Winter entstehen, da im Sommer durch die größere Schmelztätigkeit der Gletscher hellere Absätze sich bildeten. In einem See sind die Verhältnisse auf die Tätigkeit der Organismen zurückzuführen, die im Sommer ihr größtes Optimum haben, wodurch die sommerlichen Niederschläge im See durch größere Mengen von organischen Resten eine dunkle Färbung erhalten. Im Winter dagegen gedeihen die Diatomeen gut, deren absinkende Panzer hell sind. Auch die Ansichten über die grüne, braune und weiße Gur bedürfen einer eingehenden Nachprüfung.
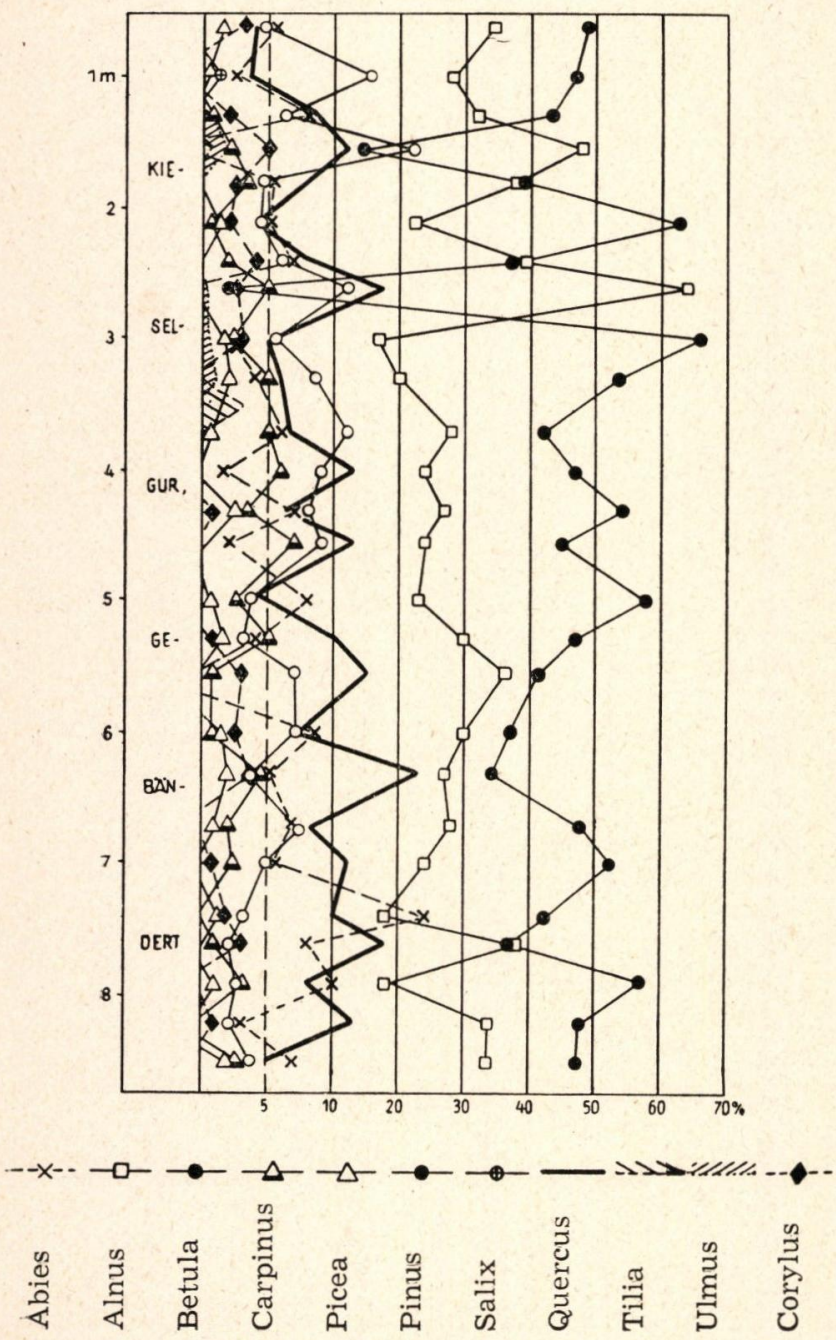

Abb. 4: Abgekürztes Pollendiagramm von Munster I.
Wie bereits oben angeführt wurde, sind die Ablagerungen der letzten Warmzeit klimatisch bedingt. Ähnlich werden die verschiedenen Gurfärbungen die

Entwicklungsphasen des Interglazials repräsentieren. Die weiße Gur ist vermutlich nicht sekundär durch Auslaugung der dunklen Bestandteile gebildet, sondern ist der Niederschlag einer kalten Zeit, in der außer den Diatomeen wenig andere Organismen gedeihen konnten (vergl. DEWALL 1929, GISTL 1928 und SOERGEL 1937).

Da GISTL die Tannenpollen nicht von denen der Fichte getrennt gezählt hat, waren neue Untersuchungen notwendig, die zu dem überraschenden Ergebnis führten, daß die Fichte neben der Tanne nur eine geringe Beteiligung besaß. Dadurch erhalten die Pollendiagramme von Munster (Abb. 4) und 
Ober-Ohe eine große Ähnlichkeit mit der Kiefern-Fichten-Tlannenzeit des Honerdinger Typus, so daß der Schluß nahe lag, beide zu parallelisieren. Die lange Zeitdauer des Interglazials schließt diese Annahme aber aus. Klieken zeigt ein ähnliches Polenbild wie Munster und Ober-Ohe, so daß infolge der weiten Verbreitung lokale Trübungen unwahrscheinlich sind. Mit den Diagrammen der PaludinenSchichten (HECK 1930), von Angerburg (KRAUSE und GROSS 1941) und Olszewice (TRELA 1929) haben sie das durchgehende Vorkommen des Tannenpollens gemeinsam. Da diese Diagramme aber eine größere waldgeschichtliche Differenzierung besitzen, stehen einer Parallelisierung große Bedenken entgegen.

Eine hohe Fichtenfrequenz in der ersten Hälfte der Warmzeit weisen ebenfalls die Ablagerungen von Ummendorf auf (SELLE 1941), deren Einordnung in die Elster-Saale-Interglazialzeit durch die geologischen Verhältnisse gesichert erscheint. Eichenberg, Northeim (HECK 1929), Zweidorf (WOLDSTEDT 1930) und Helgoland (FIRBAS 1928) haben eine gewisse Ähnlichkeit mit Ummendorf. Das Fehlen der Tannen in diesen Pollendiagrammen läßt sich vielleicht aus ihrer westlichen Lage erklären. Wegen der großen Probenabstände können gesicherte Schlüsse nicht gezogen werden, so daß wir es vorläufig mit diesen Hinweisen bewenden lassen müssen.

\section{$\mathrm{Sch}$ if t t u m}

DEWALL, W. V.: Geologisch-biologische Studie über die Kieselgurlager der Lüneburger Heide. - Jb. preuß. geol. L.-A. 49, 1929.

DYAKOWSKA, J.: Interglacial in Poniemun near Grodno (Poln. mit engl. Zusfssg.). Polska Akad. Umiejetn., Stanunia Nr. 14. Krakau 1936.

FIRBAS, F.: Über die Flora und das interglaziale Alter des Helgoländer Süßwassertöcks. - Senkenbergiana, 10. 1928.

GIESENHAGEN, K.: Kieselgur als Zeitmaß für eine Interglazialzeit. - Z. f. Gletscherkd. 14. 1926.

GISTL, R.: Die letzte Interglazialzeit der Lüneburger Heide, pollenanalytisch betrachtet. - Bot. Archiv 21, 1928.

HECK, H. L.: Pollenanalytische Untersuchungen altdiluvialer Tone und Torfe von Northeim und Eichenberg im Flußgebiet der Leine. - Jb. preuß. geol. L.-A. 49, II, 1929. - Zur Fossilführung der Berliner Paludinenschichten, ihre Beschaffenheit und Verbreitung. - Z. deutsch. geol. Ges. 82, 1930. - Die Eemund ihre begleitenden Junginterglazial-Ablagerungen bei Oldenbüttel in Holstein. - Abh. preuß. geol. L.-A., N. F. 140. Berlin 1932.

JESSEN, K. und MILTHERS, V.: Stratigraphical and Paleontological Studies of Interglacial Fresh-Water Deposits in Jutland and Northwest Germany. - Danm. Geol. Undersög., II. Raekke, Nr. 48. Kopenhagen 1928.

KRAUSE, P. G. und GROSS, H.: Das Interglazial von Angerburg nebst Bemerkungen über einige andere ostpreußische Interglaziale. - Jb. Reichsst. f. Bodenforschg. 60, 1941.

PIECH, K.: Das Interglazial in Szczercow (östlich von Wielun, Wojewodschaft Lodz). Rocznik Polsk. Tow. Geol. 8, II, 1932.

REIN, U.: Die Vegetationsentwicklung des Interglazials von Lehringen. - Z . deutsch. geol. Ges. 90, 1938.

RUST, GRIPP, SCHUTRUMPF: Das altsteinzeitliche Rentierjägerlager Meiendorf. Neumünster 1937.

SCHUTTRUMPF, R.: Das Interglazialprofil von Lauenburg a. d. Elbe (Kuhgrund II) im Lichte der Pollenanalyse. - Mitt. geol. Staatsinst. Hamburg, 16, 1937.

SELLE, W.: Beiträge zur Mikrostratigraphie und Paläontologie der nordwestdeutschen Interglaziale. - Jb. Reichsst. f. Bodenforschg. 60, 1941. - Interglaziale Süßwassermergel- und Kieselgur-Vorkommen in Niedersachsen. - N. Arch. f. Landes- und Volkskd. von Niedersachsen, H. 3, 1947.

SOERGEL, W.: Die Vereisungskurve. - Berlin 1937.

STARK, P., FIRBAS, F. und OVERBECK, F.: Die Vegetationsentwicklung des Interglazials von Rinnersdorf in der östl. Mark Brandenburg. - Abh. Nat. Ver. Bremen 28, 1932. 
STOLLER, J.: Geologisch-agronomische Karte der Gegend östlich von Verden a. d. Aller nebst Erläut. - Pr. geol. Landesanst. Berlin 1910.

TRELA, J.: Interglacial in Samostrzelniki bei Grodno in Polen. - Polska Akad. Umiej., Starunia Nr. 9, Krakau 1935. - Pollen Analysis of the interglacial formations in Olszewice. - Spraw. Kom. Fizjogr. Pol. Akad. Um. 64, Krakau 1929.

WEBER, C. A.: Utber die fossile Flora von Honerdingen und das nordwestdeutsche Diluvium. - Abh. Nat. Ver. Bremen 13, 1896.

WOLDSTEDT, P.: Über ein Interglazial bei Zweidorf (Braunschweig). Mit einem Beitrag von K. VON BÜLOW. - Z. deutsch. geol. Ges. 82, 1930. - Über die Ausdehnung der letzten Vereisung in Norddeutschland und über die Stellung des Warthe-Stadiums in der norddeutschen Eiszeitgliederung. - Ber. Reichsamt f. Bodenforsch. Wien 1942. - Über die stratigraphische Stellung einiger wichtiger Interglazialbildungen. - Z. deutsch. geol Ges. 99, 1949.

\title{
Das Interglazial von Wallensen im Hils
}

\section{Glaziale Umlagerungen interglazialer Moore}

\author{
Von Paul W. Thom son-Liblar. Mit 3 Abb.
}

Die Hilsmulde dürfte vielleicht das schönste geologische Modell im nordwestdeutschen Raume sein. Ihre geologische Bedeutung ist aber damit nicht erschōpft. Salztektonisch bedingte Senkungserscheinungen haben hier Schichtenfolgen aus verschiedenen Abschnitten des Tertiärs und Quartärs, z. T. übereinander, in wundervoller Form erhalten.

Hier befindet sich das bekannte Braunkohlenlager. von Wallensen, über dessen Alter bis jetzt keine Klarheit herrschte. Der umgelagerte sekundäre Pollen dieser Braunkohle spielt in der Hangendschichte des Interglazials eine Rolle. Ich habe hier 2 Profile, gegen 70 Einzelproben, untersucht. Außer allen im Quartär auftretenden Pollenformen, auch dem von Fagus, der im Interglazial fehlt, Ilex, cf. Hedera u. a. tritt hier der Pollen typischer tertiärer Arten und Gattungen auf. Der Pinus cf. haploxylon-Pollen ist häufiger als der von Pinus silvestris. Nicht selten sind große pinusartige Pollenkörner, die vielleicht der Gattung Keteleeria angehören dürften, neben den von Abies und Picea vorhanden. Kleine nur $70 \mu$ große Piceapollenkörner dürften von der Picea omorica oder einer nahe verwandten Art stammen. Sehr häufig, oft vorherrschend ist der Pollen der Gattung Tsuga, sowohl der vom Tsuga canadensis-, wie der vom Tsuga heterophylla-Typus. Im ganzen lassen sich 4 Tsugaformen unterscheiden. Ich habe hier Frequenzen bis $48 \%$ beobachtet. Die Tsugaarten dürften neben den Fichten und Tannen zu den häufigsten Waldbäumen dieser Zeit gehört haben. Der sehr charakteristische Pollen von Sciadopitys ist sehr verbreitet, wie auch der vom Sequoiatypus, der wohl durch lokale Bestände bedingt (Holzfunde, bestimmt von R. KRÄUSEL) in einzelnen Proben in Massen auftreten kann. Als Pollenlieferant könnte hier auch die Gattung Cryptomeria in Betracht gezogen werden. Dasselbe gilt auch für ungflügelte, glatte Coniferenpollenkörner, die von Taxodiaceen, wie Taxodium u. a., und von Cupressineen, wie Thuja, Chamaecyparis $u$. a. herrühren dürften. Von tertiären Laubbäumen tritt hier der Nyssapollen in mindestens 2 Formen in einzelnen Proben mit Frequenzen von 25\% und mehr auf, die wohl durch Lokalbestände bedingt sind. Regelmäßig ist auch der Pollen von Liquidambar anzutreffen. Dasselbe gilt auch für Carya und besonders für Pterocarya, während der von Juglans sehr selten ist. Nur ganz zerstreut sind Pollenformen von Castanea u. a. anzutreffen. Die typischen miozänen Leitformen fehlen praktisch. 